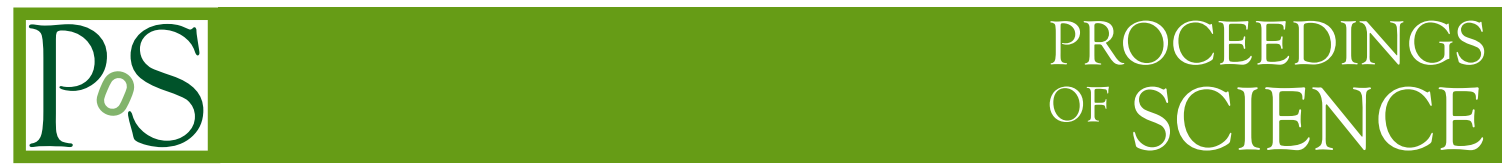

\title{
NPR determination of quark masses from the HISQ action
}

\author{
Andrew T. Lytle* \\ SUPA, School of Physics and Astronomy \\ University of Glasgow, Glasgow, G12 8QQ, UK \\ E-mail: Andrew. Lytle@glasgow.ac.uk
}

\section{HPQCD Collaboration}

I report on a calculation of bilinear Z-factors needed for determining $Z_{m}$ using non-perturbative renormalization (NPR) on $n_{f}=2+1+1$ HISQ ensembles. RI/MOM and RI/SMOM schemes are studied. These will provide an independent determination of quark masses in addition to other methods being used by the HPQCD collaboration.

The 33rd International Symposium on Lattice Field Theory

14 -18 July 2015

Kobe International Conference Center, Kobe, Japan

${ }^{*}$ Speaker. 


\section{Introduction}

Knowledge of renormalized quark mass parameters has been improved significantly in recent years from lattice QCD methods. Precise determination of these are important for BSM phenomenology and the search for new physics. For example, precision measurements of Higgs couplings to $b$ and $c$ quarks at future colliders must be combined with similarly precise SM mass determinations in order to detect deviations from the SM [1]. Currently, uncertainties in $b$ and $c$ masses are at the percent to few-percent level. Reducing uncertainties to the sub-percent level is a challenge which requires input from multiple lattice groups, as well determinations from multiple methods. Both of these help ensure a robust estimate of uncertainties which may stem from systematics in either formulation or method.

There have been several recent lattice determinations of charm mass from different groups, which are broadly in agreement with one another (a recent summary of these can be found in [2]). The most precise of these at present come from the "current-current correlator" method $[3,4]$ in simulations using a Highly Improved Staggered Quark (HISQ) action, which allows a relativistic treatment of charm, on $n_{f}=2+1$ and $n_{f}=2+1+1$ ensembles, plus continuum QCD perturbation theory through $\mathscr{O}\left(\alpha_{s}^{3}\right)$. Results from an application of this method using domain-wall fermions were also presented at this conference [5]. A precise value for $m_{c}$ can be translated into precise $m_{s}, m_{u d}$ values using bare quark mass ratios [6].

The $c, s, u d$ masses can also be obtained from $n_{f}=2+1+1$ HISQ simulations using nonperturbative renormalization (NPR) methods. RI/MOM results on $n_{f}=2+1$ asqtad ensembles were presented in [7], and in an ongoing determination with HYP-smeared valence quarks [8, 9]. Here we present first results for both RI/MOM and RI/SMOM schemes using the HISQ action. Sec. 2 describes the basic methodology with Secs. 2.2 and 2.3 presenting more details on the RI/MOM and RI/SMOM schemes respectively.

\section{NPR method}

The $\overline{\mathrm{MS}}$ renormalized quark mass is related to the bare lattice input mass $m_{0}$,

$$
m^{\overline{\mathrm{MS}}}(\mu)=Z_{m}^{\overline{\mathrm{MS}}}(\mu, 1 / a) m_{0} .
$$

In practice it is difficult to compute the factor $Z_{m}^{\overline{\mathrm{MS}}}(\mu, 1 / a)$ beyond $\mathscr{O}\left(\alpha_{s}\right)$ using lattice perturbation theory, especially with improved actions $\left(Z_{m}\right.$ was calculated to $\mathscr{O}\left(\alpha_{s}^{2}\right)$ using the asqtad action in [10]).

An alternative method was proposed in [11], which breaks the problem into two steps. The first step makes use of an intermediate Regularization Independent (RI) scheme that is well-defined both on the lattice and in the continuum. Using this scheme the renormalization conditions are imposed directly on lattice correlation functions. In this way one obtains $Z$-factors to all orders in $\alpha_{s}$, however this determination will be sensitive to discretization errors as well as non-perturbative effects. Therefore the method requires that the renormalization condition be applied within a range

$$
\Lambda_{\mathrm{QCD}} \ll|p| \ll \frac{\pi}{a} .
$$

This ensures both discretization effects and non-perturbative contributions are small. 
The second step then uses a conversion factor, computed using continuum perturbation theory, to convert the $Z$-factor to the $\overline{\mathrm{MS}}$ scheme. The continuum conversion factors are generally known to higher order than the lattice to continuum factors. It should be noted results obtained from the first step are universal and the second step is only necessary if one requires results in a non-RI scheme such as $\overline{\mathrm{MS}}$.

\subsection{Calculation details}

Results presented here were calculated on a single coarse $(a \approx 0.12 \mathrm{fm}) n_{f}=2+1+1$ HISQ ensemble [12], with lattice volume $L^{3} \times T, L=20, T=64$, and with sea masses of $a m_{c}=0.635$, $a m_{s}=0.0509$, and $a m_{l}=0.0102$. The configurations are fixed to Landau gauge. Propagator inversions were with valence masses $a m_{\mathrm{val}}=0.0509,0.0102$, and 0.00501 .

Inversions were done using momentum sources, and with a variety of momentum values. For the RI/MOM results the momenta either have the form $2 \pi(x / L, x / L, x / L, 3 x / T)$ for $x=1,2,3$ or $(x, x, x, 0)$. The latter were computed using twisted boundary conditions and are not restricted to lattice Fourier modes. RI/SMOM results have $p_{1}=2 \pi(x / L, 0, x / L, 0)$ and $p_{2}=2 \pi(x / L,-x / L, 0,0)$ with $x=3,4,5$.

\subsection{RI/MOM scheme}

We calculate off-shell Landau gauge-fixed Green functions of bilinear operators with external quark states, these have the form

$$
G_{\Gamma}^{i j}(p)=\left\langle q^{i}(p)\left(\sum_{x} \bar{q}(x) \Gamma q(x)\right) \bar{q}^{j}(-p)\right\rangle_{\mathrm{amp}} .
$$

The $i$ and $j$ indices represent both spin and color. The renormalization factors are obtained by requiring that an appropriate trace of the correlation function in the interacting theory equal its tree-level value.

$$
\Lambda_{\Gamma}(p) \equiv \frac{1}{12} \operatorname{Tr}\left[\Gamma G_{\Gamma}(p)\right] \simeq \frac{Z_{q}(p)}{Z_{\Gamma}(p)}
$$

The RI and $\overline{\mathrm{MS}}$ schemes satisfy $Z_{m}=Z_{S}^{-1}=Z_{P}^{-1}$, so that with the wavefunction renormalization factor $Z_{q}$ it is possible to obtain $Z_{m}$ from the scalar and pseudoscalar correlators. $Z_{q}$ may be obtained from the momentum-space (polespace) propagator itself.

$$
Z_{q}^{\prime}\left(p^{2}\right)=-\frac{i}{12 N_{T}} \sum_{\mu} \frac{p_{\mu}^{\prime}}{\overline{p^{\prime 2}}} \overline{\overline{\left(\gamma_{\mu} \otimes 1\right)}} S^{-1}\left(p^{\prime}\right)
$$

(The polespace propagator assumes a continuum-like form but with $N_{T}=4$ taste degrees of freedom, details may be found in [13].) In principle $Z_{m}$ could be obtained from the trace of the inverse propagator, but this quantity also contains a quark condensate contribution which is significant for momenta satisfying Eq. (2.2). This condensate also strongly affects $\Lambda_{P}$, causing it to differ from $\Lambda_{S}$ especially at low momentum where it is not suppressed.

Some results for $\Lambda_{S}$ and $\Lambda_{P}$ are shown in Fig. 1. $\Lambda_{P}$ exhibits strong mass dependence due to the condensate term, especially in the infrared, while $\Lambda_{S}$ has much milder behavior. These two quantities are compared more directly in Fig. 3 (left), and it is evident that they approach one another at large $p^{2}$ where the condensate term is suppressed. 
Results for $Z_{q}$ are shown in Fig. 2. This quantity has almost no visible mass dependence. The filled points were computed using twisted boundary conditions, which allow for continuous variation of $|p|$ keeping the orientation of $p$ fixed [14]. This is useful for combining results from different lattice spacings and gives smooth curves as a function of $(a p)^{2}$ because the lattice momenta all belong to the same hypercubic representation. These points have a different momenta orientation from the others in Fig. $2((\mathrm{x}, \mathrm{x}, \mathrm{x}, 0)$ vs. $(\mathrm{x}, \mathrm{x}, \mathrm{x}, 3 \mathrm{x}))$ and so give some indication of the size of hypercubic artifacts.
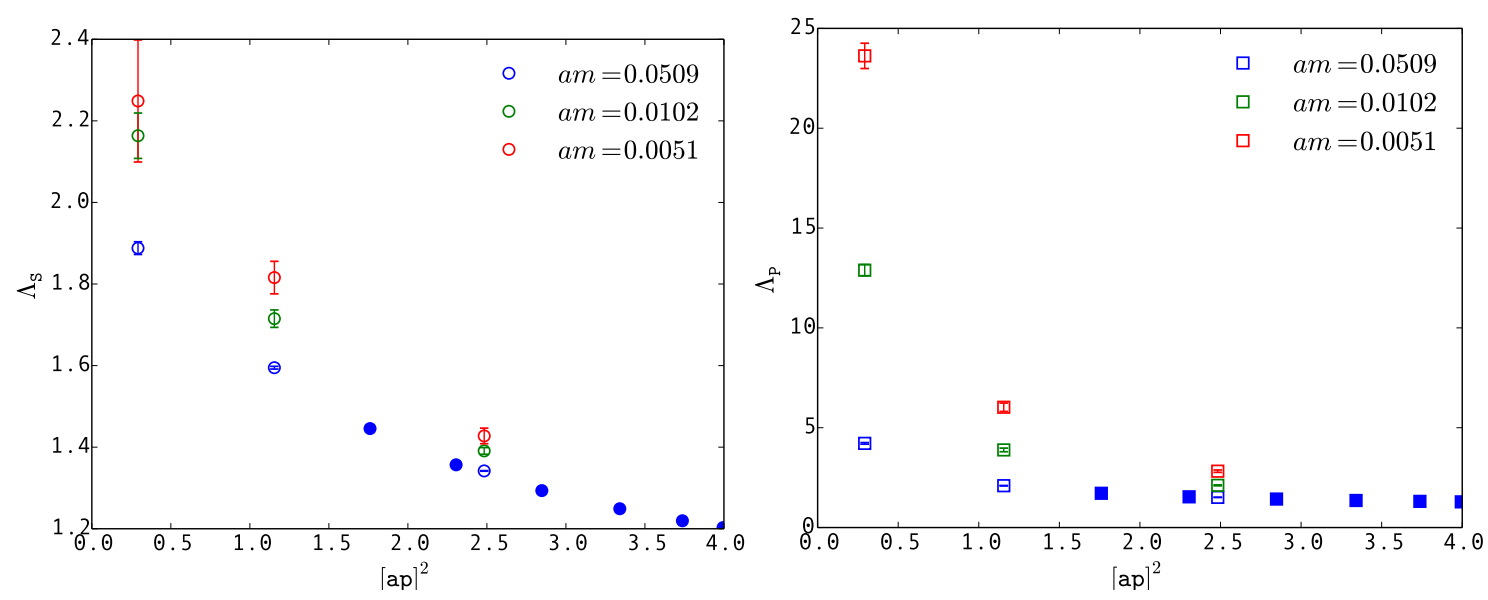

Figure 1: [Note different y-axis scales left vs. right] (Left) $\Lambda_{S}$ at three valence masses and for a range of momenta. The filled points were obtained using twisted boundary conditions. (Right) $\Lambda_{P}$ for the same masses and momenta.

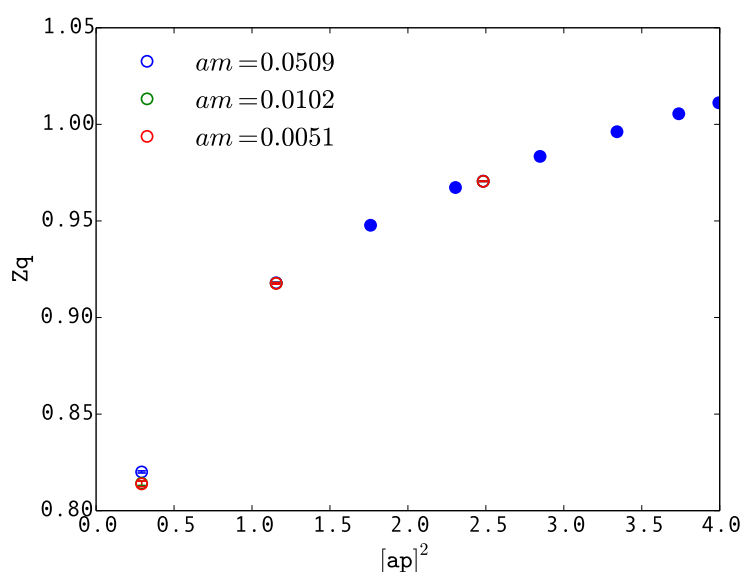

Figure 2: $Z_{q}^{\prime}$ extracted from the staggered polespace propagator at three masses. The filled points were obtained using twisted boundary conditions.

\subsection{RI/SMOM scheme}

Due to infrared sensitivity exhibited by the MOM scheme, it may be preferable to extract $Z_{m}$ using RI/SMOM intermediate schemes first formulated in [15]. SMOM schemes have several advantages: 
- Significantly reduced infrared sensitivity.

- Reduction in mass dependence of $Z_{S, P}$ observed.

- Matching factors to $\overline{\mathrm{MS}}$ scheme closer to 1 .

The SMOM scheme uses a different kinematic setup than the MOM scheme. Whereas in the MOM scheme there is a single momentum $p$ (see Eq. (2.3)), the SMOM scheme uses separate momenta $p_{1}, p_{2}$ on each leg with $p_{1}-p_{2}$ inserted at the vertex. Furthermore these momenta satisfy the constraint $p_{1}^{2}=p_{2}^{2}=\left(p_{1}-p_{2}\right)^{2}$ to maintain a single renormalization scale. The MOM and SMOM setups are also referred to as "exceptional" and "non-exceptional" respectively.

Results for $\Lambda_{S}$ and $\Lambda_{P}$ obtained from the SMOM setup at a single valence mass are shown in Fig. 3 (right). Now $\Lambda_{P}$ is much closer to $\Lambda_{S}$ over the full $(a p)^{2}$ range and especially at large $(a p)^{2}$ indicating effective suppression of the condensate contribution. This is shown further in Fig. 4 (left) which plots the difference between $\Lambda_{P}$ and $\Lambda_{S}$ over their average for both the exceptional and non-exceptional schemes. Whereas the exceptional scheme shows an $\mathscr{O}(10 \%)$ difference even at the highest momenta studied the non-exceptional difference is at most a few-percent and sub-1\% at large momentum.
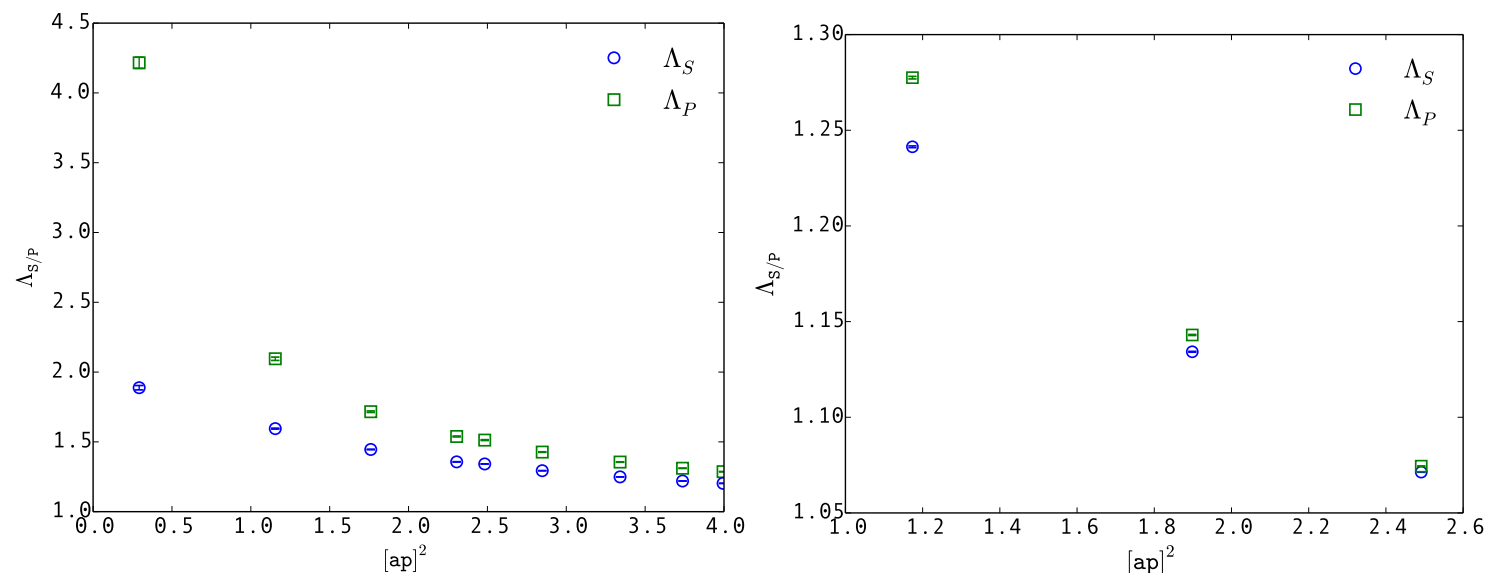

Figure 3: [Note different y-axis scales left vs. right] (Left) Comparison of $\Lambda_{S}$ and $\Lambda_{P}$ as a function of $(a p)^{2}$ for $a m_{\mathrm{val}}=0.0509$ using the exceptional scheme. (Right) Same comparison but using the non-exceptional scheme.

Fig. 4 (right) compares the mass dependence of $\Lambda_{S}$ at fixed momentum in the exceptional and non-exceptional schemes. There is a rather strong mass dependence in the exceptional case. For the non-exceptional scheme the variation is slight, from valence mass at the physical strange down to $m_{s} / 10$. This is significant in practice because the lattice results are obtained with non-zero masses and extrapolated to the chiral limit.

\section{Conclusions}

We have presented initial results for calculations of $Z_{m}$ using NPR techniques on $n_{f}=2+$ $1+1$ HISQ ensembles. We studied both exceptional (RI/MOM) and non-exceptional (RI/SMOM) 

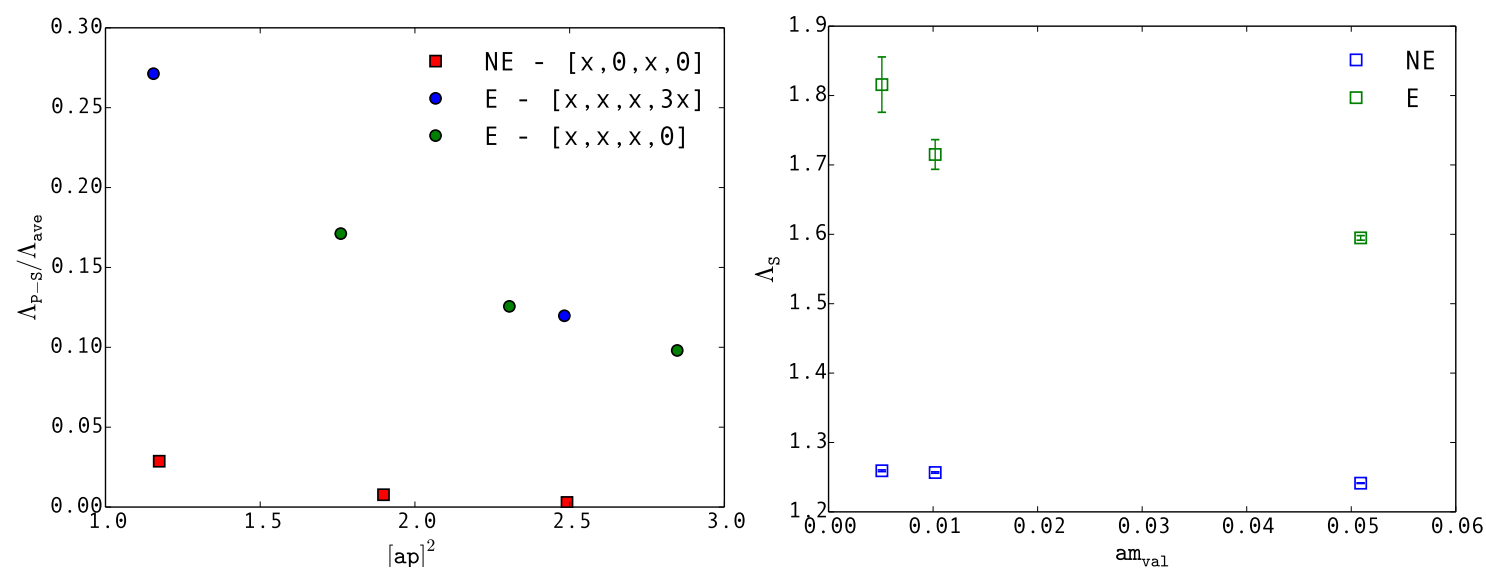

Figure 4: (Left) Difference of $\Lambda_{P}$ and $\Lambda_{S}$ divided by their average for $a m_{\mathrm{val}}=0.0509$ using exceptional (blue and green circles) and non-exceptional (red squares) kinematics. (Right) $\Lambda_{S}$ at fixed $(a p)^{2} \approx 1.2$ as a function of valence quark mass for the exceptional (green squares) and non-exceptional (blue squares) schemes.

schemes. Results in the SMOM scheme exhibit decreased sensitivity to the infrared, and a significantly reduced dependence on valence mass is also observed, as compared to the MOM scheme. These features, along with a $\overline{\mathrm{MS}}$ matching factor which is close to 1 , should prove useful in precision determination of $Z_{m}$.

The calculations presented here have been limited to a single, coarse lattice ensemble. In the future we will extend the work to fine $(a \approx 0.09 \mathrm{fm})$ and superfine $(a \approx 0.06 \mathrm{fm})$ lattices. It will also be important to use ensembles with varying sea-quark masses for a given lattice spacing in order to understand the approach to the chiral limit.

\section{Acknowledgements}

I would like to thank Christine Davies and Jonna Koponen for useful discussions. Computations were carried out on the Darwin supercomputer, part of STFC's DiRAC facility.

\section{References}

[1] G. P. Lepage, P. B. Mackenzie and M. E. Peskin, arXiv:1404.0319 [hep-ph].

[2] A. T. Lytle, arXiv:1509.03078 [hep-lat].

[3] I. Allison et al. [HPQCD Collaboration], Phys. Rev. D 78, 054513 (2008) [arXiv:0805.2999 [hep-lat]].

[4] B. Chakraborty et al. [HPQCD Collaboration], Phys. Rev. D 91, no. 5, 054508 (2015) [arXiv:1408.4169 [hep-lat]].

[5] K. Nakayama, B. Fahy and S. Hashimoto, arXiv:1511.09163 [hep-lat].

[6] C. T. H. Davies et al., Phys. Rev. Lett. 104, 132003 (2010) [arXiv:0910.3102 [hep-ph]].

[7] A. T. Lytle, PoS LAT 2009, 202 (2009) [arXiv:0910.3721 [hep-lat]].

[8] J. Kim, J. Kim, W. Lee and B. Yoon, PoS LATTICE 2013, 308 (2014) [arXiv:1310.4269 [hep-lat]]. 
[9] H. Jeong, J. Kim, W. Lee, J. Pak and S. Park, arXiv:1511.00397 [hep-lat].

[10] Q. Mason et al. [HPQCD Collaboration], Phys. Rev. D 73, 114501 (2006) [hep-ph/0511160].

[11] G. Martinelli, C. Pittori, C. T. Sachrajda, M. Testa and A. Vladikas, Nucl. Phys. B 445, 81 (1995) [hep-lat/9411010].

[12] A. Bazavov et al. [MILC Collaboration], Phys. Rev. D 82, 074501 (2010) [arXiv:1004.0342 [hep-lat]].

[13] A. T. Lytle and S. R. Sharpe, Phys. Rev. D 88, no. 5, 054506 (2013) [arXiv:1306.3881 [hep-lat]].

[14] R. Arthur et al. [RBC and UKQCD Collaborations], Phys. Rev. D 83, 114511 (2011) [arXiv:1006.0422 [hep-lat]].

[15] C. Sturm, Y. Aoki, N. H. Christ, T. Izubuchi, C. T. C. Sachrajda and A. Soni, Phys. Rev. D 80, 014501 (2009) [arXiv:0901.2599 [hep-ph]]. 